\title{
Analyzing the factors that influence occult metastasis in oral tongue cancer
}

\author{
Jung-Hyun Shin ${ }^{1,2}$, Hye-Jung Yoon ${ }^{3}$, Soung-Min Kim¹, Jong-Ho Lee' ${ }^{1}$, Hoon Myoung ${ }^{1}$ \\ ${ }^{\prime}$ Department of Oral and Maxillofacial Surgery, School of Dentistry and Dental Research Institute, Seoul National University, Seoul, \\ ${ }^{2}$ Department of Oral and Maxillofacial Surgery, Dankook University Jukjeon Dental Hospital, Yongin, \\ ${ }^{3}$ Department of Oral Pathology, Dental Research Institute, School of Dentistry, Seoul National University, Seoul, Korea
}

\begin{abstract}
J Korean Assoc Oral Maxillofac Surg 2020;46:99-107)
Objectives: We accessed the various clinico-histopathological factors, and their association with occult metastasis (OM) in oral tongue squamous cell carcinoma (OTSCC).

Materials and Methods: One hundred-nine patients with OTSCC were divided into the elective neck dissection (END) group and the watchful waiting (WW) group. Age, sex, T-stage, depth of invasion and differentiation were evaluated to determine the correlation between clinico-histopathological factors and OM. For immunohistochemical analysis, paraffin-embedded blocks of 41 OTSCC specimens were examined with antibodies (VEGF-c, c-Met, and ROR1).

Results: The group with tumor thickness of oral tongue cancer $\geq 3 \mathrm{~mm}$ had higher incidence of OM than those with a thickness of $<3 \mathrm{~mm}$. The depth of invasion was statistically correlated with OM $(P=0.022)$. Immunohistochemical analysis showed that high expression of VEGF-c $(P=0.043)$, c-Met $(P=0.009)$, and ROR-1 $(P=0.003)$ were statistically correlated with OM.

Conclusion: The analysis of these clinico-histopathological and immunohistochemical factors can help to determine neck dissection in clinically negative (cN0) patients.
\end{abstract}

Key words: Tongue cancer, Elective neck dissection, Watchful waiting, Occult metastasis, Immunohistochemistry

[paper submitted 2019. 12. 12 / revised 2020. 1. 6 / accepted 2020. 1. 28]

\section{Introduction}

Tongue cancer is the most common type of oral cancer. Unlike other head and neck areas, the vascular system and the lymphatic system are well developed in the tongue. Therefore, the incidence of cervical lymph node metastasis (LNM) is high ${ }^{1}$. LNM is the most important prognostic factor for survival of head and neck cancer patients ${ }^{2,3}$. The average 5 -year survival rate is $>50 \%$ in patients without LNM, while that of patients with LNM is only $30 \%{ }^{4}$. Unfortunately, this LNM

\section{Hoon Myoung}

Department of Oral and Maxillofacial Surgery, School of Dentistry and Dental Research Institute, Seoul National University, 101 Daehak-ro, Jongno-gu, Seoul 03080, Korea

TEL: +82-2-2072-3059 FAX: +82-2-766-4948

E-mail:myoungh@snu.ac.kr

ORCID: https://orcid.org/0000-0002-9984-8479

(c) This is an open-access article distributed under the terms of the Creative Commons Attribution Non-Commercial License (http://creativecommons.org/ licenses/by-nc/4.0/), which permits unrestricted non-commercial use, distribution, and reproduction in any medium, provided the original work is properly cited.

Copyright (C) 2020 The Korean Association of Oral and Maxillofacial Surgeons. All rights reserved. often already exists when the cancer diagnosis is made. Approximately $25 \%$ of occult metastasis (OM) is too small to be detected by imaging techniques ${ }^{5}$. Approximately $20 \%$ to $50 \%$ of $\mathrm{OM}$ has been identified in oral tongue cancer patients ${ }^{1,6}$.

The treatment of patients with clinically negative (cN0) tongue cancer remains controversial. The current treatment modalities include glossectomy followed by watchful waiting (WW), and glossectomy with elective neck dissection (END). A survey performed in the United States found that there was a lack of consensus regarding the treatment of $\mathrm{cN} 0$ neck $^{7}$. A similar finding was described in a European survey in Marburg, Germany ${ }^{8}$. Because LNM is often identified before surgery, there is a need for significant clinicopathologic factors and a highly sensitive detection method, such as immunohistochemistry.

There is growing interest in the correlation between LNM and immunohistochemical (IHC) markers. Vascular endothelial growth factor (VEGF) is essential in angiogenesis and vasculogenesis. The increment of VEGF-c expression is related to the LNM in the human thyroid, lung, prostate, gastric, 
colorectal, breast cancer, and melanoma ${ }^{9-11}$. c-Met is known as MET and hepatocyte growth factor receptor (HGFR). cMet regulates the cellular processes, cell function, and tissue homeostasis in mammalian development ${ }^{12}$. In addition, c-Met can activate lymphangiogenesis, which can ultimately cause $\mathrm{LNM}^{13,14}$. ROR1 is a transmembrane protein that regulates skeletal and neuronal development, cell polarity, and cell migration $^{15,16}$. Many studies have shown that ROR1 was overexpressed in human cancers ${ }^{17-21}$.

The aim of this study is (1) define the relationship between clinicopathologic findings and OM; (2) identify a biomarker associated with OM by immunohistochemistry; and (3) apply a useful diagnostic method for selecting treatment.

\section{Materials and Methods}

\section{Clinico-histological finding}

Patients who visited and underwent surgery at Seoul National University Dental Hospital between 2000 and 2013 were included in this study. These patients were pathologically diagnosed with squamous cell carcinoma in the oral tongue area with no apparent cervical LNM in their pre-operative work up (clinical examination, magnetic resonance imaging, ultrasonography, and positron emission tomography). The patients were divided into the following two groups: the END group and the WW group. The patients in the END group received glossectomy with END. Patients in the WW group received glossectomy, followed by WW. In the END group, we evaluated for LNM after END. In the WW group, patients underwent surveillance for neck recurrence during the WW period. In the END group, OM was defined by the presence of LNM on the histopathological examination in the neck dissection specimen. In the WW group, OM was defined by neck recurrence without recurrence at the primary site. The total number of OM cases is defined by the sum of the patients with LNM in the END group, and the number of patients with neck recurrence in the WW group.

Patients undergoing radiation therapy or chemotherapy before surgery were excluded from this study. The age, sex, clinical manifestations, and survival analysis were obtained from the medical records. Staging of the primary site and cervical LNM in oral tongue cancer was classified by the American Joint Committee on Cancer (AJCC) 7th edition. For histopathologic review, 109 cases of H\&E slides were reviewed in the Department of Oral Pathology at Seoul National University Dental Hospital from 2000 to 2013. Two oral pathologists reviewed the H\&E slides and identified the depth of invasion, differentiation and T-stage. The slides were examined under an optical microscope at a final magnification of $200 \times$. This study was reviewed by the Institutional Review Board of School of Dentistry, Seoul National University (IRB No. S-D20140041).

\section{Immunohistochemistry}

Paraffin-embedded blocks of 41 cases of oral tongue cancer specimens from the Department of Oral Pathology at Seoul National University Hospital from 2000 to 2013 were examined.

A tissue microarray was made using the re-location of the tissue from the paraffin blocks. The microarray blocks were sectioned to $3 \mu \mathrm{m}$, and were transferred to the glass. IHC staining was then performed. The slides were stained with antibodies. The slides were then incubated in the oven at $60^{\circ} \mathrm{C}$ for 1 hour, deparaffinized with xylene, rehydrated by serial dilutions with alcohol $\left(72^{\circ} \mathrm{C}\right.$ for 3 minutes, 3 times $)$, and washed with tap water for 5 minutes.

The IHC analysis of VEGF-c was performed using a Bond polymer detection kit (Leica Microsystem, Seoul, Korea) with a monoclonal antibody against VEGF-c (1:500; Santa Cruz Biotechnology, Dallas, TX, USA). The IHC analysis of c-Met was performed using an Ultraview detection kit (Ventana Medical Systems, Oro Valley, AZ, USA) with a monoclonal antibody against c-Met (RTU; Ventana Medical Systems). The IHC analysis of ROR1 was performed using an Envision kit (Dako North America, Carpinteria, CA, USA) with a polyclonal antibody against ROR1 (1:200; Santa Cruz Biotechnology).

For VEGF-c, the antigen retrieval was performed at a $\mathrm{pH}$ of 6.0 (VEGF-c) using the Epitope Retrieval 1 solution (Leica Microsystem) for 20 minutes at $100^{\circ} \mathrm{C}$. The reactions were then processed using peroxidase block solution for 5 minutes to quench the endogenous peroxidase activity. The slides were then incubated with monoclonal antibodies for 15 minutes. Next, the sections were incubated with bond polymer detection kit for 8 minutes. The slides were incubated for 10 minutes with 3, 3'-diaminobenzidine (DAB) to visualize the reaction. Finally, the slides were counterstained with Mayer's hematoxylin for one minute.

For c-Met, antigen retrieval was performed at $\mathrm{pH} 8.4$ using Cell conditioning 1 (Ventana Medical Systems) for 60 minutes at $100^{\circ} \mathrm{C}$. The slides were then incubated with a monoclonal antibodies for 32 minutes at $37^{\circ} \mathrm{C}$. Afterwards, 
an ultra-wash was performed. Finally, the slides were counterstained with Mayer's hematoxylin for 4 minutes. After the counterstain, the slides were incubated for 4 minutes in Bluing reagent.

For ROR1, the antigen retrieval was done at a $\mathrm{pH}$ of 9.0 in the retrieval buffer (Dako North America) overnight at $4{ }^{\circ} \mathrm{C}$. The reactions were treated with peroxidase block solution for 5 minutes to quench the endogenous peroxidase activity. The slides were then incubated with primary antibody, followed by incubation with the labelled polymer using two sequential 30-minute incubations. Next, the slides were incubated for 10 minutes using DAB to visualize the reaction. Finally, the slides were counterstained with Mayer's hematoxylin.

A final score for VEGF-C was defined as the sum of (a) and (b), as follows: (a) the intensity of the stain ( 0 , negative; 1 , weak; 2, moderate; 3 , strong; and 4, very strong) and (b) the percentage of positive cancer cells was $0,0 \%$ of immunostained cells; $1,<25 \%$ of immunostained cells; $2,25 \%-50 \%$ of immunostained cells; $3,50 \%-75 \%$ of immunostained cells; and $4,>75 \%$ of immunostained cells. A final score $>6$ was considered as high expression $^{22}$.

The final score for c-Met was defined as the sum of (a) and (b), as follows: (a) the intensity of the stain ( 0 , none; 1 , light yellow; 2, yellow brown; and 3, brown), and (b) the percent-

Table 1. Clinicopathologic characteristics

\begin{tabular}{|c|c|c|c|c|}
\hline & & END group $(n=71)$ & WW group $(n=38)$ & $\begin{array}{c}\text { Total }(\text { END+WW }) \\
(\mathrm{n}=109)\end{array}$ \\
\hline \multirow[t]{2}{*}{ Sex } & Male & $58(81.7)$ & $23(60.5)$ & $81(74.3)$ \\
\hline & Female & $13(18.3)$ & $15(39.5)$ & $28(25.7)$ \\
\hline \multirow[t]{2}{*}{ Age (yr) } & $\geq 50$ & $44(62.0)$ & $25(65.8)$ & $69(63.3)$ \\
\hline & $<50$ & $27(38.0)$ & $13(34.2)$ & $40(36.7)$ \\
\hline \multirow[t]{6}{*}{ T stage $^{1}$} & I & $32(45.1)$ & $30(78.9)$ & $62(56.7)$ \\
\hline & II & $34(47.9)$ & $7(18.4)$ & $41(37.6)$ \\
\hline & III & $1(1.4)$ & $0(0)$ & $1(0.9)$ \\
\hline & IV & $4(5.6)$ & $1(2.7)$ & $5(4.8)$ \\
\hline & I & $32(45.0)$ & $30(79.0)$ & $62(56.9)$ \\
\hline & II-IV & $39(55.0)$ & $8(21.0)$ & $47(43.1)$ \\
\hline \multirow[t]{2}{*}{ Depth of invasion (mm) } & $\geq 3$ & $59(83.1)$ & $23(60.5)$ & $82(75.2)$ \\
\hline & $<3$ & $12(16.9)$ & $15(39.5)$ & $27(24.8)$ \\
\hline \multirow[t]{2}{*}{ Differentiation } & Well & $61(85.9)$ & $36(94.7)$ & $97(89.0)$ \\
\hline & Moderate/poor & $10(14.1)$ & $2(5.3)$ & $12(11.0)$ \\
\hline \multirow[t]{2}{*}{ Area } & Lateral & $51(71.8)$ & $35(92.1)$ & $86(78.9)$ \\
\hline & Other regions (FOM, base) & $20(28.2)$ & $3(7.9)$ & $23(21.1)$ \\
\hline
\end{tabular}

(END: elective neck dissection, WW: watchful waiting, FOM: floor of mouth)

${ }^{1}$ Staging by the American Joint Committee on Cancer (AJCC) 7th edition.

Values are presented as number (\%).

Jung-Hyun Shin et al: Analyzing the factors that influence occult metastasis in oral tongue cancer. J Korean Assoc Oral Maxillofac Surg 2020

Table 2. Relationship between clinicopathologic factors and occult metastasis

\begin{tabular}{|c|c|c|c|c|c|}
\hline & & $\begin{array}{l}\text { OM in END group } \\
(\mathrm{n}=71)\end{array}$ & $\begin{array}{c}\text { NR in WW group } \\
(\mathrm{n}=38)\end{array}$ & $\begin{array}{c}\text { OM in total } \\
(\mathrm{END}+\mathrm{WW})(\mathrm{n}=109)\end{array}$ & $P$-value \\
\hline \multirow[t]{2}{*}{ Sex } & Male $(n=81)$ & $13 / 58$ & $4 / 23$ & $17 / 81(21.0)$ & 0.438 \\
\hline & Female $(n=28)$ & $0 / 13$ & $4 / 15$ & $4 / 28(14.3)$ & \\
\hline \multirow[t]{2}{*}{ Age (yr) } & $\geq 50(n=69)$ & $9 / 44$ & $7 / 25$ & $16 / 69(23.2)$ & 0.173 \\
\hline & $<50(\mathrm{n}=40)$ & $4 / 27$ & $1 / 13$ & $5 / 40(12.5)$ & \\
\hline \multirow[t]{2}{*}{ T stage $^{1}$} & $1(n=62)$ & $2 / 32$ & $6 / 30$ & $8 / 62(12.9)$ & 0.053 \\
\hline & $2-4(n=47)$ & $11 / 39$ & $2 / 8$ & $13 / 47(27.7)$ & \\
\hline \multirow[t]{2}{*}{ Depth of invasion (mm) } & $\geq 3(\mathrm{n}=82)$ & $13 / 59$ & $6 / 23$ & $19 / 82(23.2)$ & $0.022 *$ \\
\hline & $<3(n=27)$ & $0 / 12$ & $2 / 15$ & $2 / 27(7.4)$ & \\
\hline \multirow{2}{*}{ Differentiation } & Well ( $\mathrm{n}=97)$ & $11 / 61$ & $7 / 36$ & 18/97 (18.6) & 0.698 \\
\hline & Moderate/poor $(n=12)$ & $2 / 10$ & $1 / 2$ & $3 / 12(25.0)$ & \\
\hline \multirow[t]{2}{*}{ Area } & Lateral & $8 / 51$ & $7 / 35$ & $15 / 86(17.4)$ & 0.363 \\
\hline & $\begin{array}{l}\text { Other regions } \\
\text { (FOM, Base) }\end{array}$ & $5 / 20$ & $1 / 3$ & $6 / 23(26.1)$ & \\
\hline
\end{tabular}

(OM: occult metastasis, END: elective neck dissection, NR: neck recurrence, WW: watchful waiting, FOM: floor of mouth)

${ }^{1}$ Staging by the American Joint Committee on Cancer (AJCC) 7th edition.

* Statistically significant $(P<0.05)$.

Values are presented as number only or number $(\%)$.

Jung-Hyun Shin et al: Analyzing the factors that influence occult metastasis in oral tongue cancer. J Korean Assoc Oral Maxillofac Surg 2020 
age of positive cancer cells $(0,0 \%-5 \%$ of immunostained cells; $1,6 \%-25 \%$ of immunostained cells; $2,26 \%-50 \%$ of immunostained cells; and 3, 51\%-100\% of immunostained cells). A final score $>4$ was considered high expression ${ }^{23}$.

The staining types of ROR1 were divided into three groups: 0 , no staining; 1 , low-level or low-to-moderate-level less than $50 \%$ of cancer cells; and 2, moderate-level more than 50\% of cancer cells or high-level staining of the cancer cells. The "2" group was considered high expression. In contrast, " 0,1 " groups were considered to have low expression ${ }^{18}$.

\section{Statistical analysis}

The chi-square test and Fisher's exact test association were used to evaluate the association between OM and clinicopathologic factors, and between the IHC findings and OM. The overall survival rates (OSR) were evaluated using Kaplan-Meier method and values were compared using the log-rank test. The statistical tests were performed using IBM SPSS Statistics software (ver. 23; IBM, Armonk, NY, USA). $P$-values $<0.05$ were considered statistically significant.

\section{Results}

A total of 109 patients with squamous cell carcinoma in the tongue area were included. The distribution of clinical and pathological data (sex, age, T-stage, depth of invasion, and differentiation) in the END group and WW group are listed in Table 1. Among 71 patients who received glossectomy with END, LNM was observed in 13 patients. Neck recurrence was observed in 8 patients among the 38 patients who

Table 3. Site of occult metastasis or neck recurrence

\begin{tabular}{|c|c|c|c|}
\hline \multirow{2}{*}{ Level $^{1}$} & \multirow{2}{*}{$\begin{array}{c}\text { OM in } \\
\text { END group } \\
\text { (No. of patients) }\end{array}$} & \multicolumn{2}{|c|}{ NR in WW group } \\
\hline & & No. of patients & NR time (mo) \\
\hline \multirow[t]{2}{*}{$\mathrm{I}$} & 4 & 2 & 8.2 \\
\hline & & & 14.4 \\
\hline \multirow[t]{3}{*}{ II } & 2 & 3 & 4.1 \\
\hline & & & 6.7 \\
\hline & & & 9 \\
\hline III & 4 & 0 & \\
\hline IV & 1 & 0 & \\
\hline \multirow[t]{2}{*}{$\mathrm{II}+\mathrm{III}$} & 2 & 2 & 4.6 \\
\hline & & & 8.3 \\
\hline I+III+IV & 0 & 1 & 19.2 \\
\hline
\end{tabular}

(OM: occult metastasis, END: elective neck dissection, NR: neck recurrence, WW: watchful waiting)

${ }^{1}$ Staging by the American Joint Committee on Cancer (AJCC) 7th edition.

Jung-Hyun Shin et al: Analyzing the factors that influence occult metastasis in oral tongue cancer. J Korean Assoc Oral Maxillofac Surg 2020 received glossectomy only. As a result, the incidences of $\mathrm{OM}$ in the END group and the WW were $18.3 \%$ and $21.1 \%$, respectively. The incidence of total OM was $19.3 \%$. Eighty-one of 109 patents were male and 28 were female. The mean age was $54.4 \pm 15.4$ years, ranging from 23 to 91 years.

With regard to the size of the primary tumor, LNM was identified in the following patients in the END group: 2 among 32 patients of T1; 10 among 34 patients of T2; 0 among 1 patient of T3; and 1 among 4 patients of T4. In the WW group, neck recurrence was identified in: 6 among 30 patients of T1, 2 among 7 patients of T2, 0 among 0 patients of T3, and 0 among 1 patient of T4. The patients in the T2-4 group had more OM than did those in the T1 group, although this was not statistically significant $(P=0.053)$.

In order to investigate the depth of invasion associated with OM, patients were divided into the following two groups: patients who had tumors with a thickness of $\geq 3 \mathrm{~mm}$ and patients with a thickness of $<3 \mathrm{~mm}$. The median depth of invasion in the END group was $0.77 \pm 0.56 \mathrm{~cm}$ (range, $0.1-3.3 \mathrm{~cm}$ ). The median depth of invasion in the WW group was $0.46 \pm 0.34$ cm (range, 0.1-1.5 cm).

The group with a thickness of $\geq 3 \mathrm{~mm}$ showed a higher incidence of OM than did the group with thickness of $<3 \mathrm{~mm}$. A depth of invasion $\geq 3 \mathrm{~mm}$ was statistically associated with OM $(P=0.022)$. (Table 2)

In the END group, LNM was identified in 11 patients among 61 patients with well differentiation, 2 among 8 patients with moderate differentiation, and 0 among 2 patients with poor differentiation. In the WW group, neck recurrence was identified in 7 patients among 36 patients with well dif-

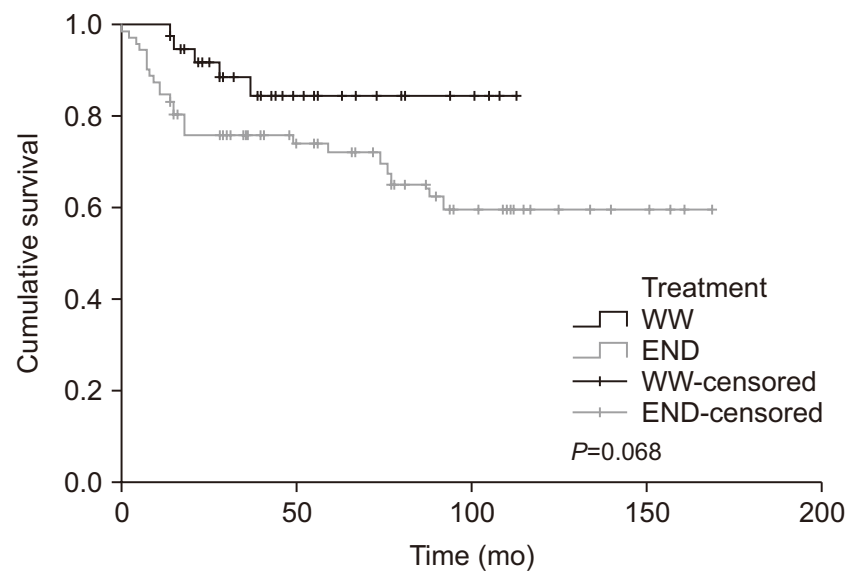

Fig. 1. Overall survival according to different treatments (KaplanMeier curves with univariate analysis: log-rank). (END: elective neck dissection, WW: watchful waiting)

Jung-Hyun Shin et al: Analyzing the factors that influence occult metastasis in oral tongue cancer. J Korean Assoc Oral Maxillofac Surg 2020 
ferentiation, 1 among 2 patients with moderate differentiation, and 0 among 0 patients with poor differentiation. The moderate/poor differentiation group had a higher incidence of OM than did the well differentiation group, although the value was not statistically significant.(Table 2)

In the END group, the incidence of OM varied depending on the primary site of the tumor. Eight of the 51 patients displayed primary tumors on the lateral surface. Four of 18 patients had primary tumors on the floor of the mouth, and one of two patients had OM on the tongue base. In the WW group, neck recurrence was found in: 7 patients among 35 patients on the lateral surface; 1 among 3 patients on the floor of mouth; and 0 among 0 patients on the tongue base. OM and neck recurrence were identified from level I through level IV. However, the values were not statistically significant. (Tables 1, 2)

The sites of recurrence on the ipsilateral side were as follows: In the END group, OM was identified in 4 patients at

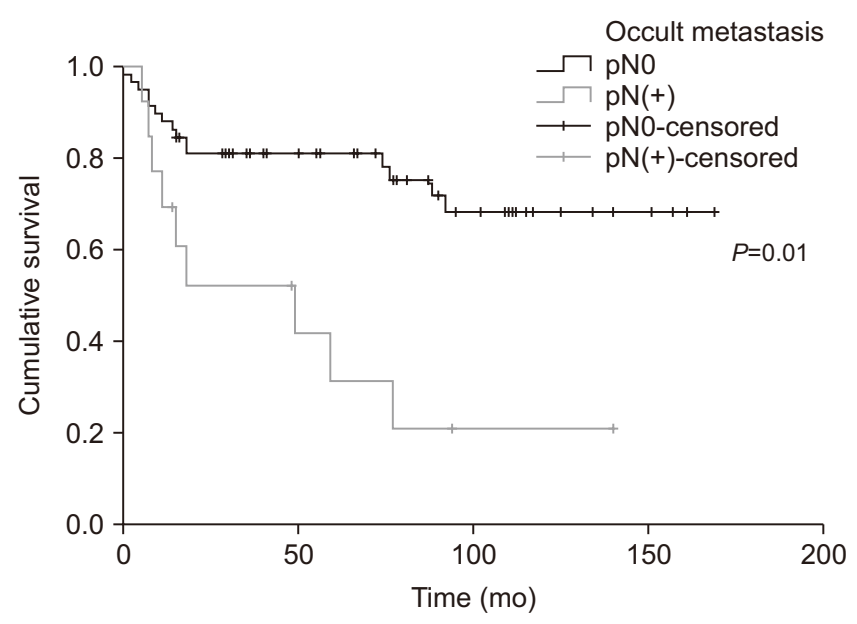

Fig. 2. Overall survival according to lymph node metastasis in elective neck dissection group (Kaplan-Meier curves with univariate analysis: log-rank).

Jung-Hyun Shin et al: Analyzing the factors that influence occult metastasis in oral tongue cancer. J Korean Assoc Oral Maxillofac Surg 2020 level I, 2 patients at level II, 4 patients at level III, and 1 patient at level IV. Two patients displayed OM at multiple levels (level II, III). In the WW group, LNM was found in 2 patients at level I, 3 patients at level II. Three patients displayed LNM at multiple levels (level II, III: 2 patients, level I, III, IV: 1 patient).(Table 3 )

The 3- and 5-year OSR in the WW group were $88.4 \%$ and $84.3 \%$, respectively. The rates in the END group were $75.8 \%$ and $71.9 \%$, respectively. The patients in the WW group had better survival rate than did those in the END group, although this difference was not statistically significant $(P=0.068)$.(Fig. 1) The 3- and 5-year OSR of the pN0 patients in the END group were $80.9 \%$ and $80.9 \%$, respectively. The OSR of the $\mathrm{pN}(+)$ group were $51.9 \%$ and $31.2 \%$, respectively $(P=0.001)$. Patients in the pN0 group demonstrated better survival rates than did those in the $\mathrm{pN}(+)$ group.(Fig. 2) The patients in the negative neck recurrence group also demonstrated better survival rates compared to those in the positive neck recurrence group. The 3- and 5-year OSR in the negative neck re-

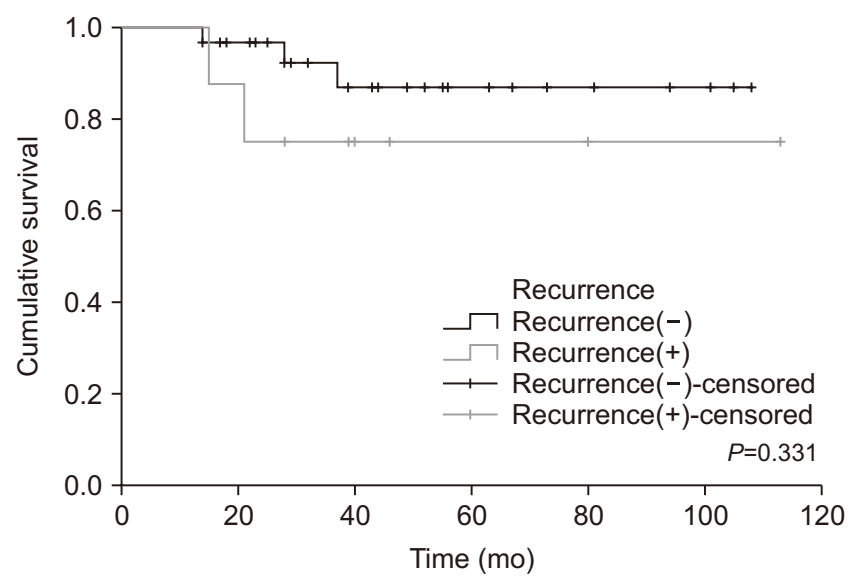

Fig. 3. Overall survival according to neck recurrence in watchful waiting group (Kaplan-Meier curves with univariate analyses: logrank).

Jung-Hyun Shin et al: Analyzing the factors that influence occult metastasis in oral tongue cancer. J Korean Assoc Oral Maxillofac Surg 2020

Table 4. Relationship between immunohistochemical factors and occult metastasis

\begin{tabular}{|c|c|c|c|c|c|c|c|c|}
\hline & & \multicolumn{2}{|c|}{ END group } & \multicolumn{2}{|c|}{ WW group } & \multicolumn{2}{|c|}{$\begin{array}{c}\text { Total } \\
(\mathrm{END}+\mathrm{WW})\end{array}$} & \multirow{2}{*}{$P$-value } \\
\hline & & $\mathrm{OM}(-)(\mathrm{n}=10)$ & $\mathrm{OM}(+)(\mathrm{n}=13)$ & $N R(-)(n=10)$ & $\mathrm{NR}(+)(\mathrm{n}=8)$ & $\mathrm{OM}(-)(\mathrm{n}=20)$ & $\mathrm{OM}(+)(\mathrm{n}=21)$ & \\
\hline \multirow[t]{2}{*}{ VEGF-c } & Low & $5(50.0)$ & $4(30.8)$ & $8(80.0)$ & $3(27.3)$ & $13(65.0)$ & $7(33.3)$ & \multirow[t]{2}{*}{$0.043^{*}$} \\
\hline & High & $5(50.0)$ & $9(69.2)$ & $2(20.0)$ & $5(71.4)$ & $7(35.0)$ & $14(66.7)$ & \\
\hline \multirow[t]{2}{*}{ c-Met } & Low & $4(40.0)$ & $1(7.7)$ & $4(40.0)$ & $0(0)$ & $8(40.0)$ & $1(4.8)$ & \multirow[t]{2}{*}{$0.009 *$} \\
\hline & High & $6(60.0)$ & $12(92.3)$ & $6(60.0)$ & $8(100)$ & $12(60.0)$ & $20(95.2)$ & \\
\hline \multirow[t]{2}{*}{ ROR1 } & Low & $7(70.0)$ & $2(15.4)$ & $6(60.0)$ & $2(25.0)$ & $13(65.0)$ & $4(19.0)$ & \multirow[t]{2}{*}{$0.003 *$} \\
\hline & High & $3(30.0)$ & $11(84.6)$ & $4(40.0)$ & $6(75.0)$ & $7(35.0)$ & $17(81.0)$ & \\
\hline
\end{tabular}

(END: elective neck dissection, WW: watchful waiting, OM: occult metastasis, NR: neck recurrence)

*Statistically significant $(P<0.05)$ by chi-square or Fisher's exact test.

Jung-Hyun Shin et al: Analyzing the factors that influence occult metastasis in oral tongue cancer. J Korean Assoc Oral Maxillofac Surg 2020 
currence group were $92.3 \%$ and $86.8 \%$, respectively. The 3and 5-year OSR in the positive neck recurrence group were $75.0 \%$ and $75.0 \%$, respectively. However, the OSR according to neck recurrence was not statistically significant $(P=0.331)$. (Fig. 3)

The IHC reactivity for VEGF-c, c-Met, Cox-2, ROR1 are summarized in Table 4.

Immunostaining for VEGF-c was detected in the cytoplasm. The images of IHC staining for VEFG-c are shown in Fig. 4. Positive VEGF-c expression was significantly correlated with OM $(P=0.043)$. Immunostaining for c-Met was detected in the cytoplasm, and the cytoplasmic membrane. Images of IHC staining for c-Met are shown in Fig. 5. Positive c-Met expression was significantly correlated with OM $(P=0.009)$. Immunostaining for ROR1 was detected in the cytoplasm, and the nucleus of cancer cells. The images of IHC staining for ROR1 are shown in Fig. 6. Positive ROR1 expression was significantly correlated with $\mathrm{OM}(P=0.003)$.

\section{Discussion}

There has been a controversy among prior retrospective studies with regard to advocating for ${ }^{6,24}$ versus opposing ${ }^{25,26}$ END. There is no consensus regarding the use of END in cN0 tongue cancer. Unfortunately, the decision to perform an END is made at the surgeon's discretion. Our study can help to guide the use of END in cN0 tongue cancer patients.

Several papers have examined the relationship between the depth of invasion and LNM. Spiro et al. ${ }^{27}$ and Brown et al. ${ }^{28}$ recommend END when the tumor thickness exceeds $2 \mathrm{~mm}$ in
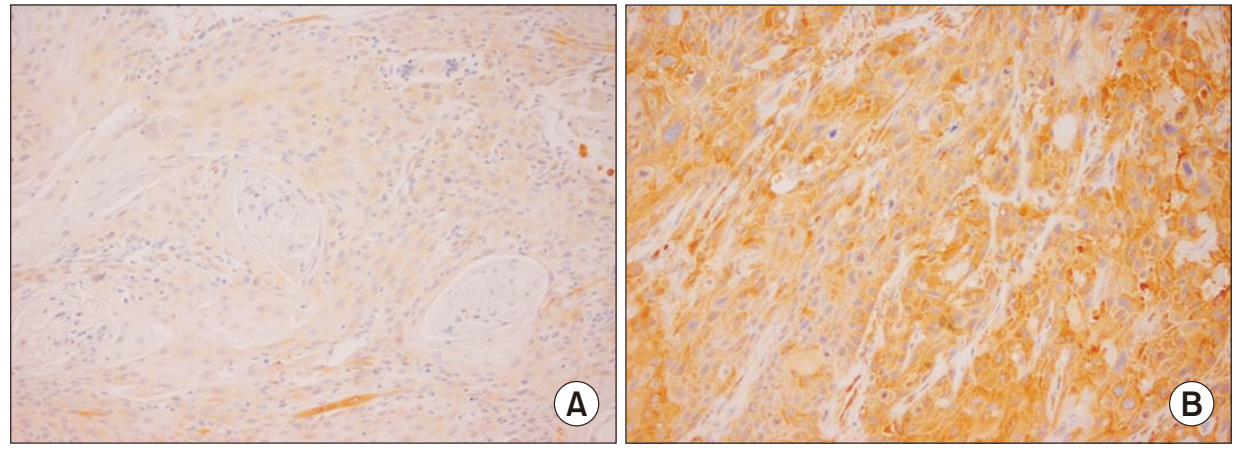

Fig. 4. Expression of VEGF-c (VEGF-C staining, $\times 200)$. A. Low expression. B. High expression.

Jung-Hyun Shin et al: Analyzing the factors that influence occult metastasis in oral tongue cancer. $J$ Korean Assoc Oral Maxillofac Surg 2020
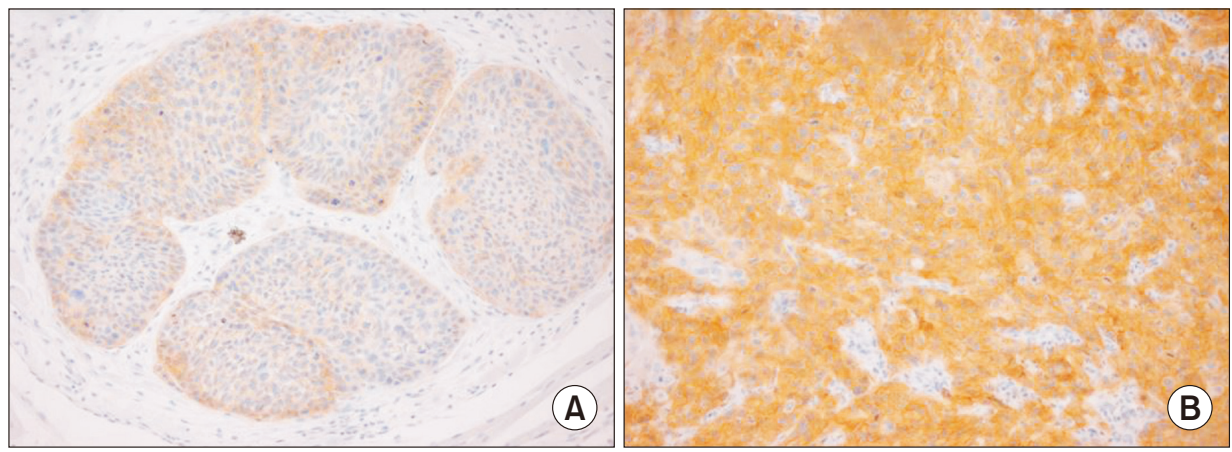

Fig. 5. Expression of c-Met (c-Met staining, $\times 200)$. A. Low expression. B. High expression.

Jung-Hyun Shin et al: Analyzing the factors that influence occult metastasis in oral tongue cancer. $J$ Korean Assoc Oral Maxillofac Surg 2020
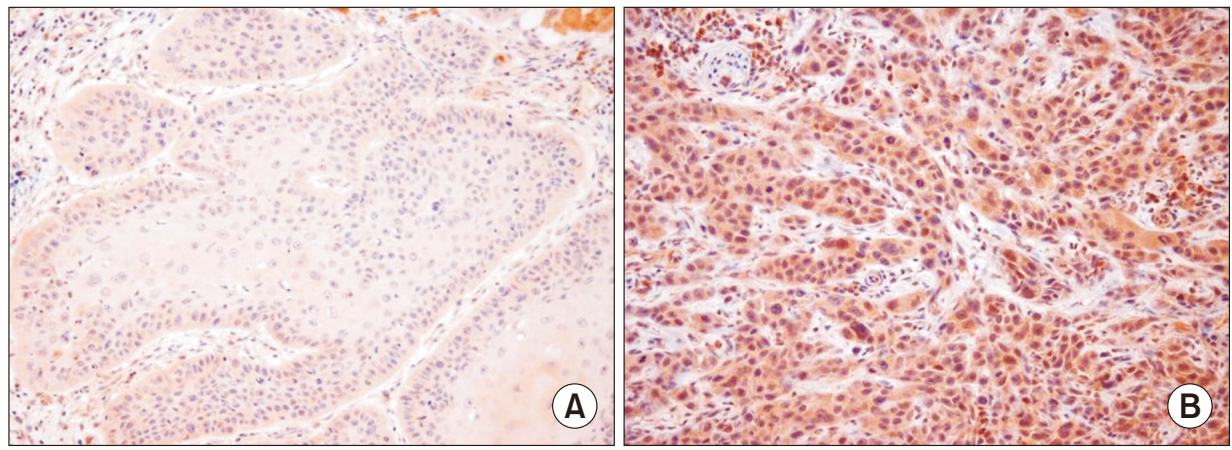

Fig. 6. Expression of ROR1 (ROR1 staining, $\times 200)$. A. Low expression. B. High expression.

Jung-Hyun Shin et al: Analyzing the factors that influence occult metastasis in oral tongue cancer. $J$ Korean Assoc Oral Maxillofac Surg 2020 
patients with oral cancer. Mohit-Tabatabai et al. ${ }^{29}$ recommend END when the tumor thickness exceeds $1.5 \mathrm{~mm}$ in patients with $\mathrm{cN} 0$ oral cancer. Our study showed that the group with a tumor thickness of $\geq 3 \mathrm{~mm}$ had a higher incidence of OM than did those with a tumor thickness $<3 \mathrm{~mm}$. Patients with a tumor thickness $\geq 3 \mathrm{~mm}$ should be treated with END. However, the exact depth of invasion may not be known until after surgery. Therefore, it is difficult to use the depth of invasion to determine whether or not END should be performed.

Some authors reported a correlation between LNM and T stage $^{27,30}$, while others found no such correlation ${ }^{31,32}$. In our study, patients in the T2-4 group demonstrated more OM than did those in the T1 group. However, there was no correlation between the T-stage and OM $(P=0.053)$.

Umeda et al. ${ }^{32}$ and Frierson and Cooper ${ }^{33}$ reported that patients with poorly differentiated tumors had a higher incidence of LNM than did those with well differentiated tumors. In contrast, we did not find a correlation between tumor differentiation and the incidence of OM $(P=0.698)$. However, patients with moderate/poor differentiated tumors showed a higher incidence of OM than did those with well differentiated tumors.(Table 2) Therefore patients with moderate/poorly differentiated tumors must be observed carefully.

There was no association between the site of tongue cancer and OM in this study. Other authors ${ }^{34,35}$ have identified similar results. Careful diagnosis is needed, particularly at the base of a mouth cancer, where cancer cells can spread to the lymph node of the contralateral neck.

The follow-up period of the WW group was 14-113 months (mean, 46.95 \pm 28.59 months). Neck recurrence in the WW group occurred in eight patients at $9.33 \pm 5.09$ months postoperatively. Neck recurrence occurred within one year in seven patients, except for one patient in the WW group. Therefore, close follow-up is necessary for one year after surgery. Multiple lymph node metastases (level I, III, IV) occurred in one patient at 14.4 months postoperatively. Neck recurrence may not have been identified during the follow-up period in this patient. Therefore, close follow-up is an important prognostic factor in the WW group.

Some studies have failed to identify any significant differences in the OSR between the END group and the WW group $^{36,37}$. In contrast, other studies demonstrated a survival benefit in the END group ${ }^{24,38}$. In this study, we found no difference between the END and WW groups. However, patients in the WW group had higher OSR than did those in the END group.(Fig. 1) There were more T1 patients than T2 patients in the WW group, while there were more $\mathrm{T} 2$ patients than $\mathrm{T} 1$ patients in the END group. It is more likely that glossectomy without neck dissection was performed in patients with $\mathrm{T} 1$ stage tumors than in patients with T2 tumors. Hiratsuka et al. ${ }^{39}$ reported that the 5-year survival rates of the patients with OM and without OM were $94 \%$ and $51 \%$, respectively. In our study, patients with $\mathrm{pN} 0$ had better survival rates than did those with $\mathrm{pN}(+)$ group in the END group.(Fig. 2)

We found that three markers that were correlated with OM were useful markers for OM detection.(Table 4)

VEGF-c expression is associated with lymphatic invasion and LNM. VEGF-c promotes lymphangiogenesis and enhances invasion via loosening of lymphatic endothelial cells $^{40}$. In this study, positive VEGF-c expression was significantly correlated with $\mathrm{OM}(P=0.043)$.

The activation of c-Met increases cancer cell proliferation, motility, invasion, LNM, and survival rates ${ }^{41-43}$. In oral squamous cell carcinoma, several studies found that overexpression of c-Met was a considerable pathologic parameter for metastasis $^{44,45}$. In this study, the positive expression of c-Met was significantly correlated with OM $(P=0.009)$. c-Met might contribute to occult metastatic processes, and facilitate the invasion of cancer cells into lymphatic vessels.

ROR1-mediated signaling has been identified in various cell lines. Wnt5a (ligand of ROR1) activates NF- $\mathrm{kB}$ in HEK293 ${ }^{46}$. Wnt5a involves the ROR1-dependent signaling pathway to enhance cancer cell growth ${ }^{18}$. In adenocarcinoma cell lines, ROR1 can phosphorylate c-SRC. EGF-induced signaling is magnified through the interaction of FZD and $\mathrm{EGFR}^{47}$. In gastric carcinoma and lung carcinoma cell lines, ROR1 is phosphorylated by MET. The silencing of ROR1 decreases cell growth ${ }^{21}$. In this study, positive ROR1 expression was significantly correlated with OM $(P=0.003)$. These markers are thought to be expressed in the early stages of LNM.

\section{Conclusion}

Our results demonstrated a relatively high incidence of OM in the OTSCC patients with $\mathrm{cN} 0$ neck tumors. We also demonstrated that the depth of invasion and IHC factors (VEGF-c, c-Met, ROR-1) are important predictive factors for the detection of OM. Surgeons can decide whether or not to perform END based on the clinical, histological, and IHC factors. Initially, close follow-up is very important. Metastatic lymph nodes in the neck can act as a source of tumor cells in there. Further prospective studies are needed to substantiate our findings. 


\section{ORCID}

Jung-Hyun Shin, https://orcid.org/0000-0002-3128-5366

Hye-Jung Yoon, https://orcid.org/0000-0002-3534-3295

Soung-Min Kim, https://orcid.org/0000-0002-6916-0489

Jong-Ho Lee, https://orcid.org/0000-0002-8843-545X

Hoon Myoung, https://orcid.org/0000-0002-9984-8479

\section{Authors' Contributions}

J.H.S. participated in data collection and wrote the manuscript. H.J.Y., S.M.K., J.H.L., and H.M. participated in the study design and performed the statistical analysis and helped to draft the manuscript. All authors read and approved the final manuscript.

\section{Ethics Approval and Consent to Participate}

This study was approved by the Institutional Review Board of School of Dentistry, Seoul National University (IRB No. S-D20140041), and the informed consent was waived.

\section{Conflict of Interest}

No potential conflict of interest relevant to this article was reported.

\section{References}

1. Ho CM, Lam KH, Wei WI, Lau SK, Lam LK. Occult lymph node metastasis in small oral tongue cancers. Head Neck 1992;14:35963.

2. Grandi C, Alloisio M, Moglia D, Podrecca S, Sala L, Salvatori P, et al. Prognostic significance of lymphatic spread in head and neck carcinomas: therapeutic implications. Head Neck Surg 1985;8:6773.

3. Snow GB, Annyas AA, van Slooten EA, Bartelink H, Hart AA. Prognostic factors of neck node metastasis. Clin Otolaryngol Allied Sci 1982; 7:185-92.

4. Whitehurst JO, Droulias CA. Surgical treatment of squamous cell carcinoma of the oral tongue: factors influencing survival. Arch Otolaryngol 1977;103:212-5.

5. van den Brekel MW, Stel HV, Castelijns JA, Nauta JJ, van der Waal I, Valk J, et al. Cervical lymph node metastasis: assessment of radiologic criteria. Radiology 1990;177:379-84.

6. Yuen AP, Wei WI, Wong YM, Tang KC. Elective neck dissection versus observation in the treatment of early oral tongue carcinoma. Head Neck 1997;19:583-8.

7. Werning JW, Heard D, Pagano C, Khuder S. Elective management of the clinically negative neck by otolaryngologists in patients with oral tongue cancer. Arch Otolaryngol Head Neck Surg 2003;129:83-8

8. Dünne AA, Folz BJ, Kuropkat C, Werner JA. Extent of surgical intervention in case of $\mathrm{N} 0$ neck in head and neck cancer patients: an analysis of data collection of 39 hospitals. Eur Arch Otorhinolaryngol 2004;261:295-303.

9. Alitalo K, Carmeliet P. Molecular mechanisms of lymphangiogenesis in health and disease. Cancer Cell 2002;1:219-27.

10. Dadras SS, Paul T, Bertoncini J, Brown LF, Muzikansky A, Jackson DG, et al. Tumor lymphangiogenesis: a novel prognostic indicator for cutaneous melanoma metastasis and survival. Am J Pathol 2003;162:1951-60.

11. Karpanen T, Egeblad M, Karkkainen MJ, Kubo H, Ylä-Herttuala S, Jäättelä M, et al. Vascular endothelial growth factor C promotes tumor lymphangiogenesis and intralymphatic tumor growth. Cancer Res 2001;61:1786-90.

12. Pennacchietti S, Michieli P, Galluzzo M, Mazzone M, Giordano $\mathrm{S}$, Comoglio PM. Hypoxia promotes invasive growth by transcriptional activation of the met protooncogene. Cancer Cell 2003;3:347-61.

13. Cao R, Björndahl MA, Gallego MI, Chen S, Religa P, Hansen AJ, et al. Hepatocyte growth factor is a lymphangiogenic factor with an indirect mechanism of action. Blood 2006;107:3531-6.

14. Kajiya K, Hirakawa S, Ma B, Drinnenberg I, Detmar M. Hepatocyte growth factor promotes lymphatic vessel formation and function. EMBO J 2005;24:2885-95.

15. Zhang H, Qiu J, Ye C, Yang D, Gao L, Su Y, et al. ROR1 expression correlated with poor clinical outcome in human ovarian cancer. Sci Rep 2014;4:5811.

16. Green JL, Kuntz SG, Sternberg PW. Ror receptor tyrosine kinases: orphans no more. Trends Cell Biol 2008;18:536-44.

17. Daneshmanesh AH, Porwit A, Hojjat-Farsangi M, Jeddi-Tehrani M, Tamm KP, Grandér D, et al. Orphan receptor tyrosine kinases ROR1 and ROR2 in hematological malignancies. Leuk Lymphoma 2013;54:843-50.

18. Zhang S, Chen L, Cui B, Chuang HY, Yu J, Wang-Rodriguez J, et al. ROR1 is expressed in human breast cancer and associated with enhanced tumor-cell growth. PLoS One 2012;7:e31127.

19. Zhang S, Chen L, Wang-Rodriguez J, Zhang L, Cui B, Frankel W, et al. The onco-embryonic antigen ROR1 is expressed by a variety of human cancers. Am J Pathol 2012;181:1903-10.

20. Cui B, Zhang S, Chen L, Yu J, Widhopf GF 2nd, Fecteau JF, et al. Targeting ROR1 inhibits epithelial-mesenchymal transition and metastasis. Cancer Res 2013;73:3649-60.

21. Gentile A, Lazzari L, Benvenuti S, Trusolino L, Comoglio PM. Ror1 is a pseudokinase that is crucial for Met-driven tumorigenesis. Cancer Res 2011;71:3132-41.

22. Faustino SE, Oliveira DT, Nonogaki S, Landman G, Carvalho AL, Kowalski LP. Expression of vascular endothelial growth factor-C does not predict occult lymph-node metastasis in early oral squamous cell carcinoma. Int J Oral Maxillofac Surg 2008;37:372-8.

23. Ma J, Ma J, Meng Q, Zhao ZS, Xu WJ. Prognostic value and clinical pathology of MACC-1 and c-MET expression in gastric carcinoma. Pathol Oncol Res 2013;19:821-32.

24. Haddadin KJ, Soutar DS, Oliver RJ, Webster MH, Robertson AG, MacDonald DG. Improved survival for patients with clinically T1/ T2, N0 tongue tumors undergoing a prophylactic neck dissection. Head Neck 1999;21:517-25.

25. White D, Byers RM. What is the preferred initial method of treatment for squamous carcinoma of the tongue? Am J Surg 1980;140:553-5.

26. O'Brien CJ, Traynor SJ, McNeil E, McMahon JD, Chaplin JM. The use of clinical criteria alone in the management of the clinically negative neck among patients with squamous cell carcinoma of the oral cavity and oropharynx. Arch Otolaryngol Head Neck Surg 2000;126:360-5.

27. Spiro RH, Huvos AG, Wong GY, Spiro JD, Gnecco CA, Strong EW. Predictive value of tumor thickness in squamous carcinoma confined to the tongue and floor of the mouth. Am J Surg 1986;152:345-50.

28. Brown B, Barnes L, Mazariegos J, Taylor F, Johnson J, Wagner 
RL. Prognostic factors in mobile tongue and floor of mouth carcinoma. Cancer 1989;64:1195-202.

29. Mohit-Tabatabai MA, Sobel HJ, Rush BF, Mashberg A. Relation of thickness of floor of mouth stage I and II cancers to regional metastasis. Am J Surg 1986;152:351-3.

30. Shaha AR, Spiro RH, Shah JP, Strong EW. Squamous carcinoma of the floor of the mouth. Am J Surg 1984;148:455-9.

31. Franceschi D, Gupta R, Spiro RH, Shah JP. Improved survival in the treatment of squamous carcinoma of the oral tongue. Am J Surg 1993;166:360-5.

32. Umeda M, Yokoo S, Take Y, Omori A, Nakanishi K, Shimada K. Lymph node metastasis in squamous cell carcinoma of the oral cavity: correlation between histologic features and the prevalence of metastasis. Head Neck 1992;14:263-72.

33. Frierson HF Jr, Cooper PH. Prognostic factors in squamous cell carcinoma of the lower lip. Hum Pathol 1986;17:346-54.

34. Ildstad ST, Bigelow ME, Remensnyder JP. Squamous cell carcinoma of the tongue: a comparison of the anterior two thirds of the tongue with its base. Am J Surg 1983;146:456-61.

35. Nason RW, Anderson BJ, Gujrathi DS, Abdoh AA, Cooke RC. A retrospective comparison of treatment outcome in the posterior and anterior tongue. Am J Surg 1996;172:665-70.

36. Yii NW, Patel SG, Rhys-Evans PH, Breach NM. Management of the N0 neck in early cancer of the oral tongue. Clin Otolaryngol Allied Sci 1999;24:75-9.

37. Duvvuri U, Simental AA Jr, D'Angelo G, Johnson JT, Ferris RL, Gooding W, et al. Elective neck dissection and survival in patients with squamous cell carcinoma of the oral cavity and oropharynx. Laryngoscope 2004;114:2228-34

38. Dias FL, Kligerman J, Matos de Sá G, Arcuri RA, Freitas EQ, Farias $\mathrm{T}$, et al. Elective neck dissection versus observation in stage I squamous cell carcinomas of the tongue and floor of the mouth. Otolaryngol Head Neck Surg 2001;125:23-9.

39. Hiratsuka H, Miyakawa A, Nakamori K, Kido Y, Sunakawa H, Kohama G. Multivariate analysis of occult lymph node metastasis as a prognostic indicator for patients with squamous cell carcinoma of the oral cavity. Cancer 1997;80:351-6.

40. Zu X, Tang Z, Li Y, Gao N, Ding J, Qi L. Vascular endothelial growth factor-C expression in bladder transitional cell cancer and its relationship to lymph node metastasis. BJU Int 2006;98:1090-3.

41. Christensen J, Anderes K. Beyond VEGF: targeting tumor growth and angiogenesis via alternative mechanisms. Adv Exp Med Biol 2008;610:43-53.

42. Gherardi E, Birchmeier W, Birchmeier C, Vande Woude G. Targeting MET in cancer: rationale and progress. Nat Rev Cancer 2012;12:89-103.

43. Cortesina G, Martone T, Galeazzi E, Olivero M, De Stefani A, Bussi M, et al. Staging of head and neck squamous cell carcinoma using the MET oncogene product as marker of tumor cells in lymph node metastases. Int J Cancer 2000;89:286-92.

44. Chen YS, Wang JT, Chang YF, Liu BY, Wang YP, Sun A, et al. Expression of hepatocyte growth factor and c-met protein is significantly associated with the progression of oral squamous cell carcinoma in Taiwan. J Oral Pathol Med 2004;33:209-17.

45. Galeazzi E, Olivero M, Gervasio FC, De Stefani A, Valente G, Comoglio PM, et al. Detection of MET oncogene/hepatocyte growth factor receptor in lymph node metastases from head and neck squamous cell carcinomas. Eur Arch Otorhinolaryngol 1997;254 Suppl 1:S138-43.

46. Fukuda T, Chen L, Endo T, Tang L, Lu D, Castro JE, et al. Antisera induced by infusions of autologous Ad-CD154-leukemia B cells identify ROR1 as an oncofetal antigen and receptor for Wnt5a. Proc Natl Acad Sci U S A 2008;105:3047-52.

47. Yamaguchi T, Yanagisawa K, Sugiyama R, Hosono Y, Shimada Y, Arima $\mathrm{C}$, et al. NKX2-1/TITF1/TTF-1-Induced ROR1 is required to sustain EGFR survival signaling in lung adenocarcinoma. Cancer Cell 2012;21:348-61.

How to cite this article: Shin JH, Yoon HJ, Kim SM, Lee JH, Myoung H. Analyzing the factors that influence occult metastasis in oral tongue cancer. J Korean Assoc Oral Maxillofac Surg 2020;46:99-107. https://doi.org/10.5125/jkaoms.2020.46.2.99 